\title{
SUPPLEMENTAL TABLE
}

Sample Name

Total Unique Productive Productive Clonality Mass of Reaction

$\operatorname{Max}$

Gene

Coverage motifs level Fig7a Fig7c Fig7d Fig7e Fig1f Fig8b Fig8c Fig8d comment

\begin{tabular}{|c|c|c|c|c|c|}
\hline 1 B6TrivaxTB10_1w_1 & 36,110 & 359 & 25,947 & 253 & 0.133 \\
\hline 2 B6TrivaxTB10_1w_2 & 21,551 & 331 & 15,423 & 231 & 0.091 \\
\hline 3 B6TrivaxTB10_1w_3 & 5,290 & 80 & 3,818 & 55 & \\
\hline & 4,298 & 70 & 3,863 & 58 & \\
\hline B6TrivaxTB10_1w_5 & 6,747 & 156 & 4,801 & 110 & \\
\hline B6TrivaxTB10 $1 w-6$ & & & & & \\
\hline B6TrivaxTB10_5wInf_1 & $1,366,057$. & 957 & $1,183,681$ & 624 & 0.528 \\
\hline 8 B6TrivaxTB10 5wInf 2 & $1,529,724$ & 1.252 & $1,137,552$ & 793 & 0.471 \\
\hline 9 B6TrivaxTB10_5wlnf_3 & $1,418,738$ & 677 & $1,018,085$ & & 0.557 \\
\hline 10 B6TrivaxTB10_5winf_4 & 491,958 & 632 & & & 0.431 \\
\hline 11 B6TrivaxTB10_5wInf_5 & 918,589 & 571 & 751,499 & 346 & 0.470 \\
\hline 12 B6TrivaxTB10_5wIIf_6 & 101,521 & 309 & 78,746 & 204 & 0.411 \\
\hline 13 aTB10prin & $1,135,007$ & 3,076 & 822,168 & 2,048 & 0.223 \\
\hline 14 aTB10prime_B8 & $1,533,180$ & & 1,099,553 & & 0.245 \\
\hline 15 aTB10prime_B9 & $1,605,354$ & 4,110 & $1,160,134$ & 2,776 & 0.226 \\
\hline 16 aTB10prime_B10 & 705,086 & 2,334 & 514,497 & 1,517 & 0.215 \\
\hline 7 Mtb4wTB10_B1 & 208,451 & 935 & 173,848 & 646 & 0.429 \\
\hline 18 Mtb4wTB10_B2 & 150,387 & 799 & 107,173 & 562 & 0.353 \\
\hline 19 Mtb4wTB10_B3 & $\begin{array}{r}3,736 \\
-770\end{array}$ & 97 & 2,809 & 69 & 0.138 \\
\hline : Mtb4wTB10_B4 & 172,707 & 822 & 132,516 & 562 & 0.408 \\
\hline & 747,497 & 1,260 & 586,252 & 828 & 0.405 \\
\hline & $\begin{aligned} 545,130 \\
127013\end{aligned}$ & 1,049 & 401,203 & & 0.300 \\
\hline 23 Mtb4wTB10_L3 & & & & & \\
\hline
\end{tabular}

$\begin{array}{rrr}84.2 & 4.3 & 869 \\ 66.9 & 2.9 & 479 \\ 78.7 & 5.0 & 95 \\ 51.8 & 4.4 & 83 \\ 112.6 & 6.2 & 200 \\ 73.6 & 16.3 & 12 \\ 364.8 & 38.2 & 27.873 \\ 40.8 & 13.5 & 27,449 \\ 400.0 & 22.1 & 38,190 \\ 32.0 & 17.0 & 6,719 \\ 320.3 & 22.8 & 5,754 \\ 283.5 & 20.1 & 5,408 \\ & & \\ 844.8 & 3.1 & 18,933 \\ 1036.8 & 1.8 & 29,133 \\ 965.8 & 1.6 & 25,362 \\ 93.0 & 4.2 & 8,829 \\ 400.0 & 16.5 & 4,536 \\ 400.0 & 14.2 & 2,958 \\ 400.0 & 5.8 & 136 \\ 400.0 & 18.7 & 4,536 \\ 40.0 & 19.5 & 17,300 \\ 400.0 & 6.3 & 13,048 \\ 400.0 & 10.0 & 2,766 \\ 400.0 & 15.9 & 9.398\end{array}$

$\begin{array}{lll}51 & \checkmark & \text { survey } \\ 58 & \checkmark & \text { survey }\end{array}$

$\begin{array}{llll}77 & \gamma & \text { survey } \\ 85 & x & \text { survey } \\ 43 & x & \text { singey }\end{array}$

$\begin{array}{lll}85 & x & \text { surve } \\ 43 & x & \text { survey } \\ 14 & x & \text { survey }\end{array}$

$56 \checkmark$ survey

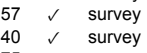

$\begin{array}{rrr}75 & x & \text { survey } \\ 171 & x & \text { survey }\end{array}$

$\begin{array}{rrr}171 & x & \text { survey } \\ 20 & x & \text { survey }\end{array}$

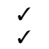

excluded because of too few sequences (blood
excluded because of too few sequences (blood)

$\checkmark$ deep

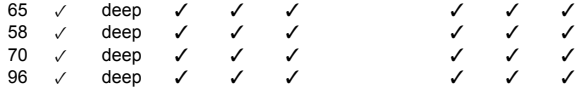

excluded because of too few sequences (from paired blood sample)
excluded because of too few sequences (from paired blood sample

excluded because of too few sequences (from paired blood sample)

$\begin{array}{rrr}57 & \text { survey } \\ 36 & \times \quad \text { survey }\end{array}$

$\begin{array}{lll}36 & \times & \text { survey } \\ 41 & \text { survey } \\ 45 & & \\ 4 & \text { surey }\end{array}$

$\begin{array}{lll}x & 2 \\ x & x\end{array}$

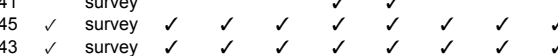

Mtb4wTB10 14

$\begin{array}{lll}127,013 & 682 & 101,000\end{array}$

$\begin{array}{ll}486 & 0.309 \\ 604 & 0.368\end{array}$

2,766
9,398

excluded because of too few sequences (blood)

excluded because of too few sequences (from paired blood sample) 